\title{
artigo
}

Andrade, C.O.; Flores, P.V.P.; Figueiredo, L.S.; Pereira, J.M.V.; Oliveira, S.X.; Calvalcanti, A.C.D.;

Construção e avaliação do jogo educativo sobre registro de enfermagem (JERE): estudo metodológico

\section{Construção e avaliação do jogo educativo sobre registro de enfermagem (JERE): estudo metodológico}

\begin{abstract}
RESUMO
Objetivo: Construir e avaliar um jogo educativo direcionado ao ensino-aprendizagem do registro de enfermagem sob a ótica do processo de enfermagem com sistemas de linguagens padronizadas. Método: 0 jogo foi construído a partir de um estudo metodológico com a participação de especialistas em processo de enfermagem e auxilio de designer. A avaliação quanto ao objetivo, organização, estilo de escrita, motivação e aparência foi realizada por 14 juízes através de um questionário validado no Brasil. Os dados foram organizados. Para verificar a concordância entre os juízes foi utilizado o índice de validação de conteúdo. Resultados: 0 jogo de tabuleiro contém 80 cartas com perguntas e respostas sobre histórico de enfermagem, diagnósticos, intervenções e resultados de enfermagem. Foi considerado totalmente adequado ou adequado quanto ao objetivo, organização, estilo de escrita, motivação e aparência com $259(71,2 \%)$ de um total de 364 dos itens avaliados. Conclusão: 0 jogo educativo sobre registro de enfermagem é uma ferramenta de ensino-aprendizagem lúdica e motivadora que pode ser utilizada para o ensino-aprendizagem do registro de enfermagem.
\end{abstract}

DESCRITORES: Enfermagem; Processos de Enfermagem; Tecnologia Educacional.

\section{ABSTRACT}

Objective: Elaborate and evaluate an educational game directed to the teaching-learning of the nursing record from the point of view of the nursing process with standardized language systems. Method: The game was constructed from a methodological study with the participation of experts in the process of nursing and assistance of designer. The evaluation of the objective, organization, writing style, motivation and appearance was performed by 14 judges through a questionnaire validated in Brazil. The data was organized. To verify the agreement between the judges, the content validation index was used. Results: The board game contains 80 letters with questions and answers about nursing history, diagnoses, interventions and nursing results. It was considered totally adequate or adequate regarding the objective, organization, writing style, motivation and appearance with 259 (71.2\%) of a total of 364 of the evaluated items. Conclusion: The educational game about nursing registration is a playful and motivational teaching-learning tool that can be used for the teaching-learning of the nursing record.

KEYWORDS: Nursing; Nursing Process; Educational Technology.

\section{RESUMEN}

Objetivo: Construir y evaluar un juego educativo dirigido a la enseñanza-aprendizaje del registro de enfermería bajo la óptica del proceso de enfermería con sistemas de lenguajes estandarizados. Método: El juego fue construido a partir de un estudio metodológico con la participación de especialistas en proceso de enfermería y ayuda de diseñador. La evaluación en cuanto al objetivo, organización, estilo de escritura, motivación y apariencia fue realizada por 14 jueces a través de un cuestionario validado en Brasil. Los datos se organizaron. Para verificar la concordancia entre los jueces se utilizó el índice de validación de contenido. Resultados: El juego de tablero contiene 80 cartas con preguntas y respuestas sobre historial de enfermería, diagnósticos, intervenciones y resultados de enfermería. Se consideró totalmente adecuado o adecuado en cuanto al objetivo, organización, estilo de escritura, motivación y apariencia con 259 $(71,2 \%)$ de un total de 364 de los ítems evaluados. Conclusión: El juego educativo sobre registro de enfermería es una herramienta de enseñanza-aprendizaje lúdica y motivadora que puede ser utilizada para la enseñanza-aprendizaje del registro de enfermería.

PALABRAS CLAVE: Enfermería; Proceso de Enfermería; Tecnología Educacional.

RECEBIDO EM: 30/04/2019 APROVADO EM: 07/05/2019

\section{Cláudia Oliveira de Andrade}

Enfermeira. Mestre pelo programa de Mestrado Profissional em Enfermagem Assistencial (MPEA) da Escola de Enfermagem Aurora de Afonso Costa (EEAAC) da Universidade Federal Fluminense (UFF). 


\section{Paula Vanessa Peclat Flores}

Doutora em Ciências Cardiovasculares pelo Programa de Pós-Graduação em Ciências Cardiovasculares (PPGCCV) da Universidade Federal Fluminense (UFF). Professora Adjunta II do Departamento de Enfermagem Médico-cirúrgica (MEM) da Escola de Enfermagem Aurora de Afonso Costa (EEAAC) da Universidade Federal Fluminense (UFF).

\section{Lyvia da Silva Figueiredo}

Enfermeira. Doutoranda em Ciências Cardiovasculares pelo Programa de Pós-Graduação em Ciências Cardiovasculares (PPGCCV) da Universidade Federal Fluminense (UFF).

\section{Juliana de Melo Vellozo Pereira}

Enfermeira. Doutoranda em Ciências Cardiovasculares pelo Programa de Pós-Graduação em Ciências Cardiovasculares (PPGCCV) da Universidade Federal Fluminense (UFF).

\section{Samara Xavier de Oliveira}

Enfermeira. Graduada pela Escola de Enfermagem Aurora de Afonso Costa (EEAAC) da Universidade Federal Fluminense (UFF).

\section{Ana Carla Dantas Cavalcanti}

Enfermeira. Professora Associada III do Departamento de Fundamentos de Enfermagem e Administração (MFE) da Escola de Enfermagem Aurora de Afonso Costa (EEAAC) da Universidade Federal Fluminense (UFF). Docente Permanente do Programa de Pós-graduação em Ciências do Cuidado em Saúde (PACCS) e do Mestrado Profissional em Enfermagem Assistencial (MPEA) da UFF. Docente colaboradora externa do Programa de Pós-graduação em Ciências Cardiovasculares da UFF. Líder do Grupo de Estudos de Sistematização da Assistência de Enfermagem da UFF (GESAE_UFF). Coordenadora da Clínica de Insuficiência Cardíaca Coração Valente.

\section{INTRODUÇÃO}

0 registro de enfermagem é uma ferramenta para organização e documentação do cuidado. Sua efetivação $m$ elhora a visibilidade e o reconhecimento profissional e representa uma possibilidade concreta de avaliação dos cuidados implemen$\operatorname{tados}^{(1-2)}$. Apesar de sua importância, este é complexo, pois envolve conhecimento científico sobre o processo de enfermagem (PE) e experiência profissional a respeito de alterações no estado de saúde de indivíduos, família e comunidade. Portanto, podem ser identificadas dificuldades para a elaboração de registros de enfermagem pautados no PE na formação e na prática profissional ${ }^{(3-4)}$.

Essas dificuldades são barreiras na documentação dos cuidados realizados e impossibilitam a avaliação dos resultados alcançados a partir de intervenções de enfermagem ${ }^{(5-9)}$. A busca de estratégias que viabilizem a elaboração de registros de enfermeiros em prontuários, manuais ou eletrônicos é um desafio para profissionais da área, pois a não elaboração de registro do PE representa a omissão de informações referentes ao julgamento clínico e tomada de decisão de enfermeiros, colocando em risco a segurança do paciente e a qualidade do cuidado prestado.

Sistemas de linguagens padronizadas (SLP) são constituídos por uma organização de conceitos que têm como finalidade a padronização de diagnósticos, intervenções e/ou resultados de enfermagem para obtenção de maior clareza, objetividade e abrangência em relação as respostas de indivíduos, famílias e/ou comunidades sobre problemas de saúde reais ou potenciais ${ }^{(6,7)}$. Alguns SLP vêm sendo utilizados no Brasil e no mundo para a padronização do registro do $\mathrm{PE}^{(6)}$, sendo os mais pesquisados a classificação de diagnósticos da NANDA-International - NANDA-I ${ }^{(8)}$, de intervenções da Nursing Intervention Classification - NIC ${ }^{(9)}$, de resultados da Nursing Outcomes Classification - $\mathrm{NOC}^{(10)} \mathrm{e}$ da International Classification Nursing Practice - ICNP ${ }^{(5,11)}$.

No entanto, estudos ${ }^{(12-15)}$ têm apontado as dificuldades para a implementação do processo de enfermagem e seu registro na prática de enfermeiros.
Muitos, inclusive, mencionam o ensino-aprendizagem na formação profissional e a continuidade da formação para o processo de raciocínio e tomada de decisão.

Um estudo ${ }^{(12)}$ realizado em São Paulo identificou as dificuldades e facilidades mencionadas por 83 enfermeiras atuantes em 20 unidades de internação de um hospital que possui o processo de enfermagem implantado na prática clínica. O diagnóstico de enfermagem e a evolução foram destacados como as fases de maior dificuldade para operacionalizar o PE. Os autores atribuíram a dificuldade ao nível de conhecimento teórico e prático sobre a temática.

Um estudo ${ }^{(13)}$ conceitual sobre a evolução do PE apontou que as dificuldades para sua implementação na prática profissional são relativas à formação profissional dos componentes da equipe de enfermagem e à organização de seu processo de trabalho, assim como às expectativas das instituições ou ambiente em que o cuidado profissional de enfermagem é realizado, ou até mesmo, o modo como os gestores da saúde entendem a Enfermagem e o papel de seus exercentes. 


\section{artigo}

Andrade, C.O.; Flores, P.V.P.; Figueiredo, L.S.; Pereira, J.M.V.; Oliveira, S.X.; Calvalcanti, A.C.D.;

Construção e avaliação do jogo educativo sobre registro de enfermagem (JERE): estudo metodológico

Outro estudo ${ }^{(14)}$ analisou alguns fatores que têm dificultado a utilização do Processo de Enfermagem em nosso país. Foram apontadas categorias de dificuldades para sua utilização inerentes à estrutura, ao cenário de ensino-aprendizagem e ao no cenário da prática assistencial.

Neste sentido, este estudo torna-se relevante por apresentar uma proposta lúdica para o ensino-aprendizagem sobre o registro do PE com uso de SLP. Trata-se de um jogo educativo que exercita a habilidade mental e aborda a temática de forma inovadora, além de entreter, entusiasmar e ensinar com maior eficiência, sendo uma estratégia facilitadora do processo ensino- aprendizagem.

$\mathrm{O}$ jogo é uma atividade rica e de grande efeito que responde às necessidades lúdicas, intelectuais e afetivas, estimulando a vida social e representando uma contribuição na aprendizagem. A motivação acontece como consequência da abordagem pedagógica adotada que utiliza a exploração livre e o lúdico. Portanto, este se apresenta como uma possibilidade de aprendizagem e construção da autoconfiança para o ensino de profissionais da área da saúde. Vem sendo considerado instrumento educativo potencialmente capaz de contribuir tanto para o desenvolvimento da educação como para a construção do conhecimento em saúde, pois é entendido como atividade divertida, estimulante, interativa, inovadora e ilustrativa ${ }^{(16)}$. $\mathrm{O}$ presente estudo tem como questão norteadora: Como seria a elaboração e avaliação de um jogo educativo direcionado ao registro de enfermagem sob a ótica do processo de enfermagem com sistemas de linguagens?

Este estudo teve como objetivo construir e avaliar um jogo educativo dire- cionado ao registro de enfermagem sob a ótica do processo de enfermagem com sistemas de linguagens padronizadas.

\section{METODOLOGIA}

Estudo metodológico que construiu e avaliou um jogo educativo para auxiliar no processo de ensino-aprendizagem sobre o registro do processo de enfermagem.

O Quadro 1 descreve as etapas utilizadas para a construção do jogo educativo. Vale ressaltar que estas foram baseadas em livro texto de Fundamentos de Enfermagem ${ }^{(17)}$ e nos livros das classificações internacionais de diagnósticos - NANDA-I ${ }^{(8)}$, intervenções - $\mathrm{NIC}^{(9)}$, resultados de enfermagem $\mathrm{NOC}^{(10)}$ e a de diagnósticos e intervenções da Classificação Internacional de Prática de Enfermagem - CIPE ${ }^{(18)}$.

Quadro 1. Etapas da construção do jogo educativo. Niterói, RJ, Brasil, 2017

\begin{tabular}{|c|c|c|c|}
\hline ETAPA & OBJETIVO & METODOLOGIA & RESPONSÁVEIS \\
\hline $\begin{array}{l}\text { 1) Reflexão e escolha } \\
\text { do tipo de jogo a ser } \\
\text { elaborado }\end{array}$ & $\begin{array}{l}\text { Discutir sobre as possibilidades } \\
\text { de jogos a serem utilizados } \\
\text { para ensino-aprendizagem do } \\
\text { registro de enfermagem. }\end{array}$ & Tempestade de ideias. & $\begin{array}{l}\text { Membros do Grupo de } \\
\text { Estudos GESAE_UFF* } \\
\text { (CNPq) }\end{array}$ \\
\hline $\begin{array}{l}\left.2^{\circ}\right) \text { Definiçãa de conte- } \\
\text { údos e elaboração das } \\
\text { regras }\end{array}$ & $\begin{array}{c}\text { Descrever as regras para a } \\
\text { utilização do JERE. }\end{array}$ & $\begin{array}{l}\text { As regras foram desenvolvidas através da } \\
\text { leitura minuciosa de regras de jogos bem } \\
\text { aceitos pelo mercado brasileiro. } 0 \text { tabuleiro } \\
\text { foi feito em acrílico rígido e as cartas } \\
\text { impressas em papel fotográfico } 180 \mathrm{~g} \text {, para } \\
\text { que tivessem o mesmo brilho de uma carta } \\
\text { de baralho. }\end{array}$ & $\begin{array}{l}\text { Membros do Grupo de } \\
\text { Estudos GESAE_UFF* } \\
\text { (CNPq) }\end{array}$ \\
\hline $\begin{array}{l}\left.3^{\circ}\right) \text { Elaboração do } \\
\text { tabuleiro }\end{array}$ & $\begin{array}{l}\text { Projetar as imagens do } \\
\text { tabuleiro e cartas do JERE. }\end{array}$ & $\begin{array}{l}\text { Foram elaboradas as imagens para } \\
\text { classificar cada grupo de perguntas. Para } \\
\text { tal, foi necessário o apoio de um designer, } \\
\text { que foi orientado a desenvolver as figuras } \\
\text { de acordo com as etapas do processo de } \\
\text { enfermagem. Foram desenvolvidas } 80 \\
\text { cartas no total geral, sendo } 20 \text { cartas de } \\
\text { histórico de enfermagem, } 20 \text { cartas de } \\
\text { diagnósticos de enfermagem, } 20 \text { cartas de } \\
\text { intervenções e } 20 \text { cartas de resultados }\end{array}$ & $\begin{array}{l}\text { Membros do Grupo de } \\
\text { Estudos GESAE_UFF* } \\
\text { (CNPq) e designer gráfico }\end{array}$ \\
\hline $\begin{array}{l}\left.4^{\circ}\right) \text { Definição de conte- } \\
\text { údo para elaboração de } \\
\text { perguntas e respostas }\end{array}$ & $\begin{array}{l}\text { Elaborar perguntas e respostas } \\
\text { para as cartas classificadas } \\
\text { em cada fase do processo de } \\
\text { enfermagem (PE). }\end{array}$ & 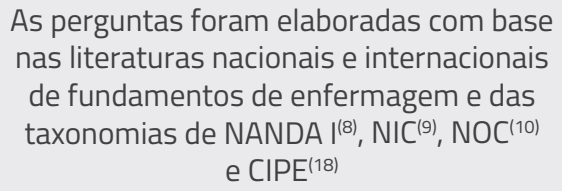 & $\begin{array}{l}\text { Membros do Grupo de } \\
\text { Estudos GESAE_UFF*(CNPq) }\end{array}$ \\
\hline
\end{tabular}

Nota: *Grupo de Estudos em Sistematização da Assistência de Enfermagem da UFF - Cadastrado no Diretório do CNPq. 
Os responsáveis pelas $1^{\mathrm{a}}$ e $4^{\mathrm{a}}$ etapas se reuniram três vezes em uma sala de reuniões do Grupo de Sistematização da Assistência de Enfermagem da Universidade Federal Fluminense (GESAE_UFF), cada reunião teve duração de duas horas para reflexão e escolha do tipo de jogo, sendo então, decidida a utilização de um jogo de tabuleiro, com uso de cartas contendo perguntas acerca da temática central.

Para a $4^{\mathrm{a}}$ etapa (elaboração de perguntas e respostas), foram realizados quatro encontros de duas horas cada. Para compor o grupo que elaborou as cartas, foram convidadas duas enfermeiras doutoras e duas enfermeiras doutorandas integrantes do GESAE_UFF, que lecionam ou pesquisam a temática de PE com SLP há mais de 5 anos.

Para o comitê de juízes, foram selecionados enfermeiros com experiência na área de PE com SLP e alunos do Curso de Graduação em Enfermagem da Escola de Enfermagem Aurora de Afonso Costa da Universidade Federal Fluminense (EEA$\mathrm{AC} / \mathrm{UFF}$ ) que já haviam concluído as disciplinas de Fundamentos de Enfermagem I e II, visto que, nestas disciplinas os alunos aprendem e desenvolvem o PE com SLP.

O comitê foi composto por 14 juízes para avaliação de conteúdo e aparência. Os juízes foram convidados através de uma carta e preencheram o Termo de Consentimento Livre e Esclarecido (TCLE) e um questionário de caracterização, contendo: sexo, idade, instituição na qual traba- lha, área de atuação, experiência anterior com $\mathrm{PE}$, participação em algum projeto de grupo de pesquisa. Formação escolar na qual se graduou, ano de conclusão da Graduação, maior titulação, se desenvolveu ou está desenvolvendo como autor ou orientador estudo na temática de PE, participação em grupos de pesquisa sobre PE, atividades profissionais e no ensino sobre a temática da PE.

O comitê de juízes foi convidado a participar de 3 rodadas do jogo e, posteriormente, a preencher o instrumento de avaliação, que é subdivido em cinco tópicos: objetivos, organização, estilo da escrita, aparência, motivação.

A avaliação dos peritos foi apresentada em tabela, com cálculos de frequência simples e percentual. Para avaliar a validade de conteúdo do JERE, utilizou-se o Índice de Validade de Conteúdo (IVC), com um índice de concordância mínimo de $70 \%$ a ser considerado para que o jogo fosse validado.

\section{RESULTADOS}

Trata-se de um jogo de tabuleiro com perguntas e respostas sobre registro de enfermagem pautado em situações clínicas sobre o PE com SLP. É composto por: dois dados, 20 cartas sobre histórico de enfermagem, 20 cartas sobre diagnóstico de enfermagem, 20 cartas sobre intervenções de enfermagem e 20 cartas sobre resultados de enfermagem.
A identidade visual do jogo foi estabelecida através das iniciais JERE, que explicitam Jogo Educativo Registro de Enfermagem, e foi distribuído de tal forma que indique que a sistematização de enfermagem é um processo contínuo e interligado.

A maioria dos juízes é do sexo feminino (71,4\%), com média de idade de 26,4 anos, trabalha em instituição pública $(57,1 \%)$, atua em área hospitalar (50,0\%), possui experiência prévia com $\mathrm{PE}(100 \%)$ e participa em grupo de pesquisa $(71,4 \%)$. São graduados em Enfermagem em instituição pública (64,3\%), com tempo de formação médio de 4,0 anos. A maioria tem ou teve experiência de atuação em PE durante Graduação $(71,4 \%)$, no entanto, não apresentam experiência com docência sobre o assunto (78,4\%).

A Tabela 1 descreve a avaliação do jogo de acordo com o julgamento dos 14 juízes. Quanto ao conteúdo do JERE, das 364 respostas (26 questões multiplicadas pelo número de juízes), 194 (53,3\%) foram consideradas totalmente adequadas, $65(17,8 \%)$ adequadas, 59 (16,2\%) parcialmente adequadas e apenas $46(12,6 \%)$ foram consideradas inadequadas. Somando os julgamentos considerados totalmente adequado e adequado, totalizou-se 259 pontos, com IVC geral de $71,1 \%$, apresentando uma concordância acima de $70 \%$. No entanto, como demonstrado a seguir nas análises parciais, os únicos blocos com IVC>70\% foram os referentes à aparência e motivação (blocos 4 e 5, respectivamente).

Tabela 1. Julgamento dos peritos sobre o JERE em relação aos itens objetivos, organização, estilo da escrita, aparência e motivação. Niterói, RJ, Brasil, 2017 ( $n=14$ )

\section{CRITÉRIOS DE AVALIAÇÃO}

$N(\%)$

\section{TOTALMENTE ADEQUADO/} ADEQUADO

PARCIALMENTE ADEQUADO

INADEQUADO

\section{Objetivos}

Atende aos objetivos

Ajuda durante o trabalho

Está adequada para ser utilizado

Total 1

\section{Organização}

O jogo é atraente e indica o conteúdo

O tamanho está adequado

$\begin{array}{ccc}8(57,1) & 3(21,4) & 3(21,4) \\ 11(78,6) & 1(7,1) & 2(14,3) \\ 6(42,9) & 6(42,9) & 2(14,3) \\ 25(59,5) & 10(23,8) & 7(16,7) \\ 10(71,4) & & \\ 9(64,3) & 2(14,3) & 2(14,3) \\ & 4(28,6) & 1(7,1)\end{array}$




\section{artigo}

Andrade, C.O.; Flores, P.V.P.; Figueiredo, L.S.; Pereira, J.M.V.; Oliveira, S.X.; Calvalcanti, A.C.D.

Construção e avaliação do jogo educativo sobre registro de enfermagem (JERE): estudo metodológico

\begin{tabular}{|c|c|c|c|}
\hline Os tópicos tem sequência & $9(64,3)$ & $3(21,4)$ & $2(14,3)$ \\
\hline Há coerência entre as informações & $10(71,4)$ & $3(21,4)$ & $1(7,1)$ \\
\hline O material é apropriado & $10(71,4)$ & $3(21,4)$ & $1(7,1)$ \\
\hline O número de cartas é suficiente & $10(71,4)$ & $3(21,4)$ & $1(7,1)$ \\
\hline $\begin{array}{l}\text { Os termos retratam aspectos impor- } \\
\text { tantes }\end{array}$ & $9(64,3)$ & $1(7,1)$ & $4(28,6)$ \\
\hline Total parcial 2: & $67(68,4)$ & $19(19,4)$ & $12(12,2)$ \\
\hline \multicolumn{4}{|l|}{ Estilo da escrita } \\
\hline O conteúdo da escrita está adequado & $10(71,4)$ & $3(21,4)$ & $1(7,1)$ \\
\hline O texto é inerente ao conteúdo & $11(78,6)$ & $1(7,1)$ & $2(14,3)$ \\
\hline O vocabulário é acessível & $11(78,6)$ & $2(14,3)$ & $1(7,1)$ \\
\hline Associação das cartas com PE & $10(71,4)$ & $2(14,3)$ & $2(14,3)$ \\
\hline O texto é claro & $3(21,4)$ & $11(78,6)$ & - \\
\hline A redação se aplica ao público - alvo & $11(78,6)$ & - & $3(21,4)$ \\
\hline Total parcial 3: & $56(66,7)$ & $19(22,6)$ & $9(10,7)$ \\
\hline \multicolumn{4}{|l|}{ Aparência } \\
\hline $\begin{array}{l}\text { As cartas e tabuleiro estão organizados } \\
\text { de acordo com a SAE }\end{array}$ & $11(78,6)$ & $1(7,1)$ & $2(14,3)$ \\
\hline As ilustrações são simples & $11(78,6)$ & - & $3(21,4)$ \\
\hline As ilustrações complementam os textos & $10(71,4)$ & $1(7,1)$ & $3(21,4)$ \\
\hline As ilustrações são expressivas & $11(78,6)$ & $1(7,1)$ & $2(14,3)$ \\
\hline Total parcial 4: & $43(76,8)$ & $3(5,4)$ & $10(17,8)$ \\
\hline \multicolumn{4}{|l|}{ Motivação } \\
\hline O material é apropriado & $11(78,6)$ & - & $3(21,4)$ \\
\hline O conteúdo tem lógica & $11(78,6)$ & $1(7,1)$ & $2(14,3)$ \\
\hline Os textos sugerem ações & $12(85,7)$ & $2(14,3)$ & - \\
\hline Aborda assuntos cotidianos & $12(85,7)$ & $2(14,3)$ & - \\
\hline Instiga mudanças de comportamento & $11(78,6)$ & $2(14,3)$ & $1(7,1)$ \\
\hline Propõe conhecimento & $11(78,6)$ & $1(7,1)$ & $2(14,3)$ \\
\hline Total parcial 5: & $68(81)$ & $8(9,5)$ & $8(9,5)$ \\
\hline Total geral: & $259(71,2)$ & $59(16,2)$ & $46(12,6)$ \\
\hline
\end{tabular}

Nota: *Cálculo de frequência simples (percentual).

O bloco de perguntas sobre o critério de avaliação referente ao objetivo e metas do JERE possui 03 subitens para pontuação máxima de 42 ( 5 itens X 14 peritos) para sua validação. Destas 42 opções de respostas, 25 foram para totalmente adequado e adequado, obtendo-se um IVC parcial 1 de 59,5\%.

O bloco de perguntas 2 apresenta as respostas dos peritos quanto à forma de apresentar as orientações do JERE. Possui sete itens, totalizando pontuação máxima de 98 (7 itens X 14 peritos) para sua validação, sendo que 67 pontos foram referentes aos julgamentos de totalmente adequado e adequado, obtendo um IVC parcial 2 de $68,3 \%$.

O bloco de perguntas 3 traz a avaliação dos peritos quanto às características linguísticas, compreensão e o estilo da escrita do JERE. Possui seis itens, fazendo com que a pontuação máxima para sua validação seja igual a 84 (6 itens $\mathrm{X}$ 14 peritos). Das 84 opções de respostas, 56 foram referentes aos julgamentos totalmente adequado e adequado, obtendo um IVC parcial 3 de 66,6\%.

O bloco de perguntas 4 traz a análise pelos peritos das características que avaliam a aparência JERE. Possui quatro 
itens, sendo que a pontuação máxima para sua validação é igual a 56 (4 itens X 14 peritos). Das 56 opções de respostas, 43 foram referentes aos julgamentos totalmente adequado e adequado, obtendo um IVC parcial 4 de $76,7 \%$.

O bloco de perguntas 5 mostra a análise dos peritos quanto à capacidade do material em causar algum impacto, motivação e/ou interesse, assim como ao grau de significação do material educativo apresentado. Possui seis itens, com pontuação máxima para sua validação igual a 84 (6 itens X 14 peritos). Das 84 opções de respostas, 68 foram referentes aos julgamentos totalmente adequado e adequado, obtendo um IVC parcial 5 de $80,9 \%$.

\section{DISCUSSÃO}

Este estudo apresentou pela primeira vez um jogo educativo sobre o registro de enfermagem utilizando os SLPs ${ }^{(5,11)}$, tais como $\mathrm{NNN}^{(8-10)}$ e CIPE ${ }^{(18)}$, com o intuito de facilitar o ensino-aprendizagem de graduandos de enfermagem e enfermeiros em educação permanente e, consequentemente, subsidiar a melhoria na qualidade dos registros de enfermagem.

O JERE foi considerado pela maior parte dos juízes como uma tecnologia que poderá ajudar os profissionais em suas atividades cotidianas. No caso de enfermeiros, este poderá ser utilizado em treinamentos em serviço e/ou cursos de capacitação. Para graduandos de enfermagem, o jogo poderá ser utilizado como uma ferramenta educativa importante em sala de aula, para induzir através de perguntas e respostas o pensamento crítico e motivar a tomada de decisão clínica pautadas no processo de enfermagem.

O pensamento crítico estabelece uma relação entre as etapas do processo diagnóstico em enfermagem. Portanto, as aplicações do processo de enfermagem, no ensino e na prática clínica, reafirmam um caminho para o raciocínio clínico $^{(19)}$. O termo raciocínio clínico

\section{O JERE foi conside-} rado pela maior parte dos juízes como uma tecnologia que poderá ajudar os profissionais em suas atividades cotidianas. No

caso de enfermeiros, este poderá ser utili-

zado em treinamen-

tos em serviço e/ou

cursos de capacitação.

Para graduandos de

enfermagem, $o$ jogo

poderá ser utilizado

como uma ferramenta

educativa importante

em sala de aula, para

induzir através de

perguntas e respostas

o pensamento crítico

e motivar a tomada de

decisão clínica pau-

tadas no processo de

enfermagem. é utilizado para designar os processos mentais envolvidos no atendimento aos usuários dos sistemas de saúde. Este se desenvolve, dentre outros fatores, a partir dos conhecimentos científicos e profissionais. O JERE é uma tecnologia educativa que pode aprimorar o pensamento crítico e raciocínio clínico dos enfermeiros por integrar, através de um jogo de tabuleiro, situações clínicas que unem respostas humanas psicossociais a respostas humanas fisiológicas das pacientes sob situações de cuidado por enfermeiros.

Distintos produtos educativos têm sido produzidos para diversos públicos e suas necessidades, apontando a riqueza de oportunidades para o cuidado educativo, como cartilhas, blogs e jogos $^{(20)}$. O JERE foi considerado interativo e propõe a busca ativa de conhecimento, pois apresenta abordagem direcionada aos assuntos cotidianos da prática de acadêmicos de enfermagem e enfermeiros. Os objetivos do jogo foram aceitos pelo comitê de juízes, $57,1 \%$, consideraram o mesmo adequado e/ou totalmente adequado.

Não ficou claro para os juízes que o jogo era direcionado apenas a profissionais de enfermagem e/ou graduandos de enfermagem. Apesar do JERE permitir a utilização de outros profissionais, seu intuito é facilitar a aprendizagem sobre registro de enfermagem com uso de SLP. Esta avaliação se reafirma quando apenas $42,8 \%$ consideram que o JERE pode ser utilizado. Portanto, ainda são necessários ajustes no jogo para que o mesmo possa ser utilizado como uma ferramenta lúdica de ensino-aprendizagem. As tecnologias devem medir a reflexão dos indivíduos para mudanças em seus comportamentos, pois estes influenciam de forma direta na saúde-doença ${ }^{(5)}$.

Quanto aos textos das perguntas e respostas das cartas, os juízes fizeram uma avaliação negativa. Nas sugestões expressas em texto individual, estes argumentaram que existem situações clínicas muito extensas, dificultando o 


\section{artigo}

Andrade, C.O.; Flores, P.V.P.; Figueiredo, L.S.; Pereira, J.M.V.; Oliveira, S.X.; Calvalcanti, A.C.D.;

Construção e avaliação do jogo educativo sobre registro de enfermagem (JERE): estudo metodológico

raciocínio clínico durante as partidas do jogo e com um certo grau de competição. Nas cartas iniciais, tinham as perguntas as respostas e as respostas certas eram escritas de vermelho. Foi realizada alteração no formato do gabarito das cartas que deixou de ter a resposta no corpo da carta para ficar em outra carta com todas as respostas, essa carta possui 80 números e cada número com a letra correspondente do gabarito de cada carta.

Ao percebermos a importância do registro de enfermagem e dos sistemas de linguagens padronizadas, considerou-se pertinente a construção e validação de uma tecnologia educativa que problematizasse a temática junto aos acadêmicos de enfermagem e enfermeiros. A validação de tecnologias mostra-se fundamental, visto que, por meio desse processo, proporciona-se aos instrumentos maior confiabilidade, subsidiando, assim, práticas e pesquisas voltadas para a área da enfermagem $^{(21)}$.

\section{CONCLUSÃO}

O JERE é uma ferramenta importante para o ensino, para a prática as- sistencial de enfermagem, bem como para o desenvolvimento de novas pesquisas, pois representa um produto lúdico para facilitar o ensino-aprendizagem de PE, subsidiando a melhoria da qualidade dos registros de enfermagem, melhorando a qualidade da assistência de enfermagem. Para que o JERE possa ser utilizado com graduandos de enfermagem e enfermeiros, torna-se necessário seu aprimoramento até que todos os itens sejam considerados adequados pelos juízes, assim como, a validação de conteúdo e de face com experts de enfermagem e designers gráficos.

\section{REFERÊNCIAS}

1. Blair W, Smith B. Nursing documentation: frameworks and barriers. Contemp Nurse. 2012; 41(2):160-8.

2. Royal College of Nursing. Abbreviations and other short forms in patient/clinic records. United Kingdon: RCN; 2012

3. Silva GS, Santos RM, Crispim LM, Almeida LMWS. Conteúdo dos registros de enfermagem em hospitais: contribuições para o desenvolvimento do processo de enfermagem. Enferm. Foco. 2016; 7 (1): 24-27.

4. Carneiro SM, Dutra HÁ, Costa FM, Mendes SE, Arreguy-Sena C. Uso de abreviaturas nos registros de enfermagem em um hospital de ensino. Rev Rene. 2016 mar-abr; 17(2):208-16.

5. Silva ERR, Cavalcanti ACD, Caldas MCRG, Lucena AF, Almeida MA, Linch GFC, Silva MB, Staub MM. Advanced Nursing Process Quality: Comparing the International Classification for Nursing Practice (ICNP) with the NANDAInternacional (NANDA-I) and Nursing Interventions Classification (NIC). J Clin Nurs. 2017 Feb; 26(3-4):379-387.

6. Carvalho EC, Cruz DALM, Herdman HT. Contribuição das linguagens padronizadas para a produção do conhecimento, raciocínio clínico e prática clínica da Enfermagem.Rev. bras. enferm. 2013 Sep; 66(spe):134-141.

7. Peres HHC, Jensen R, Martins TYC. Avaliação da acurácia diagnóstica em enfermagem: papel versus sistema de apoio à decisão. Acta Paul Enferm. 2016; 29(2):218-24.

8. Herdman TH, Kamitsuru S. Diagnósticos de Enfermagem da NANDA: Definições e classificação 2015-2017. 10th ed. Porto Alegre: Artmed; 2015;

9. Bulecheck GM, Dotcherman JM, Butcher HK. NIC: Classificação das Intervenções de Enfermagem. 5th ed. Rio de Janeiro: Elsevier; 2010;

10. Moorhead S, Johnson M, Maas ML, Swanson E. NOC: Classificação dos Resultados de Enfermagem. 4th ed. Rio de Janeiro: Elsevier; 2010.
11. Garcia TR, Nobrega MML. Processo de Enfermagem: Da teoria à prática assistencial e de pesquisa. Esc enferm Anna Nery. 2009; 13(1):816-818.

12. Takahashi AA, Barros ALBL, Michel JLM, Souza MF. Dificuldades e facilidades apontadas por enfermeiras de um hospital de ensino na execução do processo de enfermagem. Acta paul. Enferm. 2008; 21(1):32-8.

13. Maria MB, Maria CBD. Obstáculo para implementação do processo de enfermagem no Brasil. Revista enfermagem UFPE online. 2007 jul.-set.; 1(1):95-99.

14. Ribeiro CM. Auditoria de serviços de enfermagem. Rev Bras Enferm. 1972 jan/abril; 25(4):91-103.

15. Sasso MKD, Pereira SRCC, Maria GC. Revisão integrativa: método de pesquisa para a incorporação de evidências na saúde e na enfermagem. Texto contexto - enferm. 2008 Dec; 17(4): 758-764.

16. Zacharias VL. 0 Jogo Simbólico. 2002. Disponível em: http://members.tripd.com./Ifcamara/ojogona.html.

17. Potter PA, Perry AG. Grande tratado de enfermagem prática. São Paulo (SP):Tempo; 1996.

18. Mazoni SR, Rodrigues CC, Santos DS, Rossi LA, Carvalho EC. Classificação Internacional para a Prática de Enfermagem e a Contribuição Brasileira. Rev. Bras. Enferm. 2010 Mar.-Apr.; 63 (2): 1-6.

19. Bittencourt GKGD, Crossetti MGO. Theoretical model of critical thinking in diagnostic processes in nursing. Online braz j nurs. 2012 0ct; 11(Suppl 1): 563-7.

20. Mariano RM, Pinheiro AKB, Aquino OS, Ximenes Ib, Pagliuca LMF. 0 jogo educativo na promoção da saúde de adolescentes: revisão integrativa. Rev Eletr Enf. 2013;15(1):265-73.

21. Valli GP, Cogo ALP. School blogs about sexuality: an exploratory documentary study. Rev Gaúcha Enferm. 2013; 34(3):31-7. 